\title{
Prescrições geriátricas inapropriadas e polifarmacoterapia em enfermarias de clínica médica de um Hospital-Escola
}

\author{
Inappropriategeriatricprescriptionsand poly-phamacotherapyinnedal diricverosta \\ UniversityHbspital
}

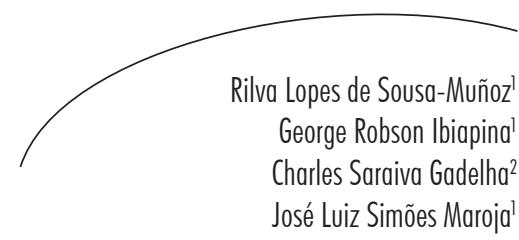

Resumo

Objetivos: Avaliar a prevalência do uso de prescrições potencialmente inapropriadas (PPI) e polifarmacoterapia na clientela idosa hospitalizada nas enfermarias de clínica médica do Hospital Universitário Lauro Wanderley (HULW), Universidade Federal da Paraíba. Metodologia: Estudo observacional e transversal com análise das prescrições geriátricas realizadas por médicos residentes em clínica médica no HULW. A amostra foi composta pela totalidade dos pacientes idosos internados nas enfermarias do HULW entre maio de 2007 e maio de 2008. Para identificar PPI, foram adotados os Critérios de BeersFick. Definiu-se polifarmacoterapia como o uso de mais de cinco medicamentos por prescrição. Resultados: Avaliaram-se as prescrições de 79 pacientes de 60 a 94 anos $(70,4$ $+/-7,3), 59,5 \%$ do sexo masculino. O número de medicamentos usados variou de 2 a 12 $(6,1+/-2,0)$ por paciente. As classes terapêuticas mais prescritas foram as relacionadas aos aparelhos digestório (83,6\%), cardiovascular (63,9\%) e nervoso (49,2\%). Os princípios ativos mais prescritos foram metoclopramida, ranitidina, dipirona e captopril. A maioria dos pacientes, 36 (59\%), apresentou polifarmacoterapia. Mais da metade dos pacientes $(54,1 \%$, ) recebeu pelo menos uma prescrição inapropriada, com maior frequência de nifedipina, butilescopolamina e diazepam. Conclusões: Identificaram-se, além de uma alta prevalência de PPI e polifarmacoterapia, excesso de prescrições de antiulcerosos e antieméticos na amostra estudada. Estes resultados indicam que maior atenção deve ser dada à prescrição para pacientes idosos. A difusão do conceito de uso inapropriado para pacientes idosos mediante educação continuada para médicos residentes poderia facilitar a adoção dos critérios de Beers-Fick, ainda não conhecidos por toda a comunidade médica.

Abstract

Objectives: To assess the prevalence of Potentially Inappropriate Prescribing (PIP) and many different drugs in hospitalized elderly client in the infirmary of medical clinic, Lauro Wanderley University Hospital (LWUH), Federal University of Paraíba. Methodology:

\footnotetext{
Curso de Medicina, Departamento de Medicina Interna. Universidade Federal da Paraíba. João Pessoa, PB, Brasil.

2 Curso de Medicina. Universidade Federal da Paraíba. João Pessoa, PB, Brasil.
}

Palavras-chave: Prescrições de Medicamentos. Saúde do Idoso. Hospitalização. Polimedicação. 
Observational and cross-sectional analysis of prescriptions geriatric performed by resident physicians in internal medicine at LWUH. The sample was composed of all elderly patients hospitalized in the LWUH between May 2007 and May 2008. The Beers-Fick criteria were used to identify PIP. Poly-pharmacotherapy was defined as the use of more than five drugs per prescription. Results: We evaluated 79 patients aged 60 to 94 years $(70.4+/-7.3), 59.5 \%$ were male. The number of medications used ranged from 2 to $12(6.1+/-2.0)$ per patient. The most prescribed therapeutic classes were due to digestive $(83.6 \%)$, cardiovascular $(63.9 \%)$ and nervous $(49.2 \%)$. The most frequently prescribed were metoclopramide, ranitidine, dipyrone and captopril. Most patients, 36 $(59 \%)$, had many different drugs. More than half of patients (54.1\%) received at least one inappropriate prescription, with a higher frequency of nifedipine, scopolamine and diazepam. Conclusions: We identified, in addition to a high prevalence of PIP and many different drugs, over-prescription anti-ulcer and anti-emetics in the sample. These results indicate that more attention should be given to prescribing for elderly patients. The diffusion of the concept of PIP for elderly patients through continuing education for medical residents could help the adoption of the Beers-Fick criteria, not yet known throughout the medical community.

\section{INTRODUÇÃO}

O fundamento deste trabalho é a preocupação atual com o uso irracional de medicamentos para pacientes geriátricos. A investigação da racionalidade da prescrição para idosos através de estudos sobre o uso de medicamentos é uma prática recomendada como ferramenta na avaliação da qualidade do serviço de saúde prestado a essa população. ${ }^{1}$ A terapêutica medicamentosa é um componente fundamental da atenção clínica ao idoso, e a otimização desta prescrição tem se tornado um importante problema de saúde em todo o mundo. A demanda deste grupo por recursos de saúde é intensa, tanto no que se refere à utilização de serviços de saúde quanto no que diz respeito ao uso de medicamentos. $^{2}$

Várias características do envelhecimento afetam a prescrição medicamentosa, tornando a seleção da farmacoterapia apropriada um processo complexo para clínicos generalistas. Os idosos consomem, em média, de dois a cinco medicamentos, e são mais sensíveis aos efeitos de certas classes farmacológicas. Isso motivou o desenvolvimento de estudos que identificaram os medicamentos inadequados para idosos ou cujo uso deve ser avaliado. ${ }^{3}$
Key words: Drug Prescriptions. Health of the Elderly. Hospitalization. Polypharmacy.
Tais estudos levaram ao desenvolvimento de listas de substâncias a serem evitadas nesses pacientes, ou usadas apenas em circunstâncias excepcionais. ${ }^{4}$ Há mais de 20 fármacos potencialmente contraindicados para os idosos, entre os quais os benzodiazepínicos e os hipoglicemiantes orais de meia-vida longa, os barbituratos de curta duração, os antidepressivos com forte ação anticolinérgica, alguns analgésicos opioides e relaxantes musculares, por exemplo. ${ }^{5,6}$

Medicamento potencialmente inadequado para o uso em idosos é definido como qualquer medicamento cujos riscos são maiores que os benefícios. Alguns autores consideram o uso desses medicamentos como a maior causa de reações medicamentosas na terceira idade. ${ }^{3}$ Portanto, compreender os padrões de utilização de medicamentos é essencial para planejar e estabelecer melhorias na atenção em saúde para o idoso. ${ }^{7}$ Sabe-se que o uso dos medicamentos varia não somente conforme idade, sexo e condições de saúde dos pacientes, mas também em função de fatores de natureza social, econômica e demográfica. ${ }^{6}$

Ainda não há estudos realizados no Estado da Paraíba focalizando este problema. O objetivo deste estudo é avaliar a prevalência do 
uso de prescrições potencialmente inapropriadas e polifarmacoterapia na clientela idosa hospitalizada nas enfermarias de clínica médica do HULW, em João Pessoa, Paraíba.

\section{METODOLOGIA}

Seguiu-se um modelo de pesquisa observacional, do tipo transversal, a partir da análise das prescrições medicamentosas de pacientes idosos atendidos nas enfermarias do Hospital Universitário Lauro Wanderley (HULW), entre maio de 2007 e setembro de 2008. Os dados foram colhidos mediante análise documental das prescrições médicas dos idosos no $10^{\circ}$ dia de internação e de entrevistas individuais com estes pacientes, com a finalidade de coletar dados sociodemográficos e registrar as medicações utilizadas antes da hospitalização. A amostra foi constituída por 79 pacientes idosos internados no referido período, que não tinham suas prescrições elaboradas pelos médicos pesquisadores. Os critérios de exclusão foram: o uso de polifarmácia ou de prescrição inapropriada antes da internação; idosos que em algum momento durante a internação tinham suas prescrições elaboradas pelos médicos pesquisadores; e negativa em participar do estudo. Foram excluídos 18 idosos que já utilizavam medicações inapropriadas ou polifarmácia antes da internação; além de três que, em algum momento, tiveram suas prescrições elaboradas pelos médicos pesquisadores. Não houve negativa em participar do estudo.

O HULW é um hospital terciário de referência em João Pessoa e cidades vizinhas, tanto da Paraíba, quanto de pequenas cidades do estado de Pernambuco e Rio Grande do Norte. Esta instituição de ensino desenvolve, além das ações de um hospital regional, procedimentos mais complexos, sendo parte da sua demanda constituída por doentes encaminhados de outros serviços de saúde da região. Suas enfermarias de clínica médica dispõem de 70 leitos. A população idosa assistida no serviço caracterizase por ser de baixa renda e desfavorável nível de instrução, representando quase $40 \%$ do total de internações no setor. ${ }^{8}$
Considerou-se prescrição inapropriada com base nos critérios de Beers-Fick e polifarmacoterapia como o uso de mais de cinco medicamentos por prescrição ao dia. ${ }^{9}$ Empregou-se a $3^{\text {a }}$ versão dos Critérios de Beers para identificar prescrições potencialmente inapropriadas (PPI) ${ }^{10}$ considerando apenas a lista de medicações inapropriadas, não levando em conta a interação medicamentos-doença. Foi mantida a metildopa - que constava na lista original de Beers, mas que foi excluída da versão modificada - por fazer parte da Relação Nacional de Medicamentos Essenciais no Brasil. Foram introduzidos também agentes laxativos catárticos na lista de medicamentos inadequados, conforme preconizam Girotto \& Silva.?

Para a determinação da prevalência da prescrição de medicamentos, foram levantados, a partir das folhas de prescrição de cada paciente idoso internado nas enfermarias de clínica médica do HULW, todos os medicamentos prescritos durante a permanência na enfermaria. Os prescritores do HULW podiam solicitar aquisição externa de medicamentos não listados na relação de medicamentos padronizados do hospital, não limitando o prescritor a uma opção de fármaco por categoria terapêutica.

O formulário de dados biodemográficos foi elaborado pelos autores e pré-testado. Os pesquisadores (três médicos residentes de Clínica Médica do HULW), previamente treinados, realizaram as entrevistas com a finalidade de coletar dados biodemográficos e registrar as medicações utilizadas antes da internação. Os pesquisadores também realizaram o registro do diagnóstico principal da internação a partir da análise dos prontuários e o registro das medicações prescritas durante a internação.

Consideraram-se idosos os pacientes com idade igual ou superior a 60 anos, de acordo com a Política Nacional de Saúde do Idoso (PNSI) do Ministério da Saúde, publicada em 1999. ${ }^{11}$

As variáveis primárias do estudo foram: (a) número de medicamentos prescritos para cada paciente; (b) polifarmacoterapia; e (c) medicamentos potencialmente inapropriados 
para idosos. As variáveis secundárias foram: (a) idade; (b) diagnóstico principal da internação; e (c) gênero.

Considerou-se classe terapêutica (ou farmacológica) o grupo de fármacos que agem sobre determinada função, tecido, órgão ou sistema orgânico conforme o sistema AnatômicoTerapêutico-Químico - ATC. ${ }^{12} \mathrm{O}$ diagnóstico principal correspondeu ao problema clínico que motivou a admissão do paciente no hospital, sendo categorizado por grupo de doenças segundo a $10^{a}$ Classificação Internacional de Doenças e Problemas Relacionados à Saúde. ${ }^{13}$

Os dados foram analisados através do programa SPSS para Windows, versão 17.0. Utilizou-se análise descritiva (porcentagens para variáveis categóricas e médias para variáveis contínuas). $\mathrm{Na}$ estatística inferencial, foram empregados os testes não-paramétricos de Mann-Whitney e o teste de qui-quadrado. O nível de significância $(p)$ adotado para os testes estatísticos foi de $5 \%$.
O projeto de pesquisa do presente estudo foi submetido à apreciação pelo Comitê de Ética em Pesquisa do HULW (protocolo no 043/07; folha de rosto $\left.n^{\circ} 13302\right)$. Todos os pacientes assinaram o termo de consentimento informado após esclarecimentos sobre a pesquisa.

\section{RESULTADOS}

Foram avaliadas as prescrições de 79 pacientes internados nas enfermarias de clínica médica do HULW no período de maio de 2007 a setembro de 2008 - portanto, no intervalo de 16 meses de observação, durante o qual os pacientes foram incluídos de forma consecutiva no estudo.

$\mathrm{Na}$ caracterização sociodemográfica da amostra, observou-se que a idade variou de 60 a 94 anos (70,4 +/- 7,3), 59,5\% do sexo masculino. A distribuição da amostra quanto ao motivo principal da hospitalização está demonstrada na tabela 1.

Tabela 1 - Motivo principal da hospitalização, categorizado por grupo de doenças segundo a $10^{\mathrm{a}}$ Classificação Internacional de Doenças e Problemas Relacionados à Saúde de pacientes idosos internados nas enfermarias de clínica médica do Hospital Universitário Lauro Wanderley. João Pessoa, PB, 2008. $(\mathrm{n}=79)$

\begin{tabular}{ccc}
\hline Motivo principal da hospitalização & $\mathrm{f}$ & $\%$ \\
\hline Doenças do aparelho digestório & 27 & 34,2 \\
Doenças do aparelho cardiovascular & 14 & 17,7 \\
Neoplasias & 9 & 11,4 \\
Doenças do sistema nervoso & 7 & 8,9 \\
Doenças do sistema respiratório & 6 & 7,6 \\
Demais categorias & 16 & $<5 \%$ (cada) \\
\hline
\end{tabular}

O número de medicamentos usados variou de dois a $12(6,1+/-2,0)$ por paciente. Os princípios ativos mais prescritos foram metoclopramida, ranitidina, dipirona e captopril. Observou-se que não houve diferença de gênero em relação ao número de medicamentos usados por prescrição $(p$ $=$ NS), mas verificou-se diferença no valor médio do número de medicamentos prescritos de acordo com a faixa etária $(p=0.04)$, com maior número de prescrições na faixa etária de 60 a 69 anos (tabela 2). 
Tabela 2 - Número de medicamentos prescritos por paciente em função de intervalos de idade da amostra de pacientes idosos internados nas enfermarias de clínica médica do Hospital Universitário Lauro Wanderley. João Pessoa, PB, 2008. (n=79)

\begin{tabular}{cccc}
\hline Classes de idade (anos) & $\mathrm{n}$ & Médias & Desvio-padrão \\
\hline $60-69$ & 45 & 6,3 & 1,7 \\
$70-79$ & 25 & 5,4 & 1,8 \\
80 ou mais & 09 & 5,4 & 1,9 \\
Amostra & 79 & 5,9 & 1,8 \\
\hline
\end{tabular}

Mais da metade dos pacientes recebeu pelo menos uma prescrição inadequada segundo os critérios de Beers (54,1\%), tendo maiores frequências a nifedipina, seguida pela butilescopolamina e o diazepam.

A maioria dos pacientes da amostra apresentou polifarmacoterapia (47/59,5\%), ou seja, uso de cinco ou mais medicamentos por prescrição médica. A prevalência desta variável não diferiu entre homens e mulheres da amostra, nem em função dos intervalos de idade considerados (60-69, 70-79 e 80 anos ou mais). O resultado no grupo etário de
60-69 anos dos pacientes (31/45\%) não alcançou significância estatística. Entre as categorias etárias de 70-79 anos e de 80 anos ou mais, o percentual de pacientes que recebeu prescrição inapropriada foi semelhante, 43 e $38 \%$, respectivamente.

De modo semelhante, observou-se correlação estatisticamente significativa entre idade e número de medicamentos prescritos por paciente, sendo tal correlação negativa (quanto maior a idade, menor o número de medicamentos prescritos) e de moderada intensidade $(r=-0,30)$, como mostra a figura 1 .

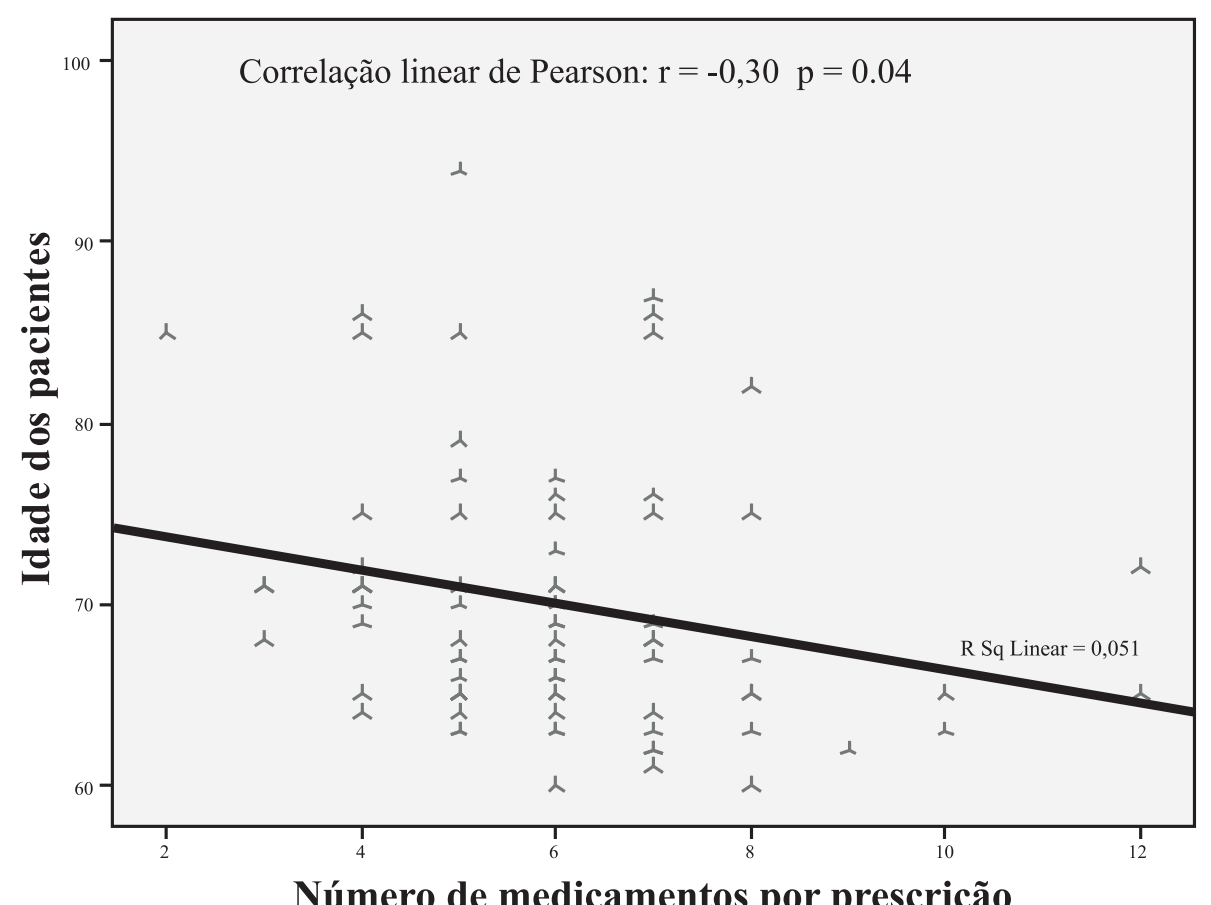

Figura 1 - Prescrição inapropriada (PI) em função da faixa de idade (números percentuais de pacientes) em pacientes idosos internados nas enfermarias de clínica médica do Hospital Universitário Lauro Wanderley. João Pessoa, PB, 2008. (n=79) 
As classes terapêuticas mais prescritas foram as relacionadas aos aparelhos digestório $(63 / 79,7 \%)$, cardiovascular (48/60,2\%) e nervoso $(31 / 39,2 \%)$, segundo a tabela 3. Dentre estas classes, os princípios ativos mais prescritos foram metoclopramida, ranitidina, dipirona e captopril. $\mathrm{O}$ uso de antibióticos foi verificado em 25,3\% dos pacientes.

Tabela 3 - Frequências de prescrição de medicamentos segundo a Classificação ATC, conforme o grupo terapêutico em pacientes idosos internados nas enfermarias de clínica médica do Hospital Universitário Lauro Wanderley. João Pessoa, PB, 2008. (n=79)

\begin{tabular}{ccc}
\hline Classes (C) de medicamentos por grupo terapêutico (ATC) & $\mathrm{f}$ & $\%$ \\
\hline C1- Digestório & 63 & 79,7 \\
C3- Cardiovascular & 48 & 60,8 \\
C9- Nervoso & 31 & 39,2 \\
C7- Antibióticos & 20 & 25,3 \\
C2- Hematopoiético & 14 & 17,7 \\
C6- Hormonais & 13 & 16,5 \\
C11- Respiratório & 5 & 6,3 \\
C8- Musculoesquelético & 4 & 5,1 \\
C4- Pele & 3 & 3,8 \\
C10- Antiparasitários & 2 & 2,5 \\
\hline
\end{tabular}

ATC: Classificação da ATC (Anatomical Therapentic Chemical), da Organização Mundial de Saúde para os estudos de utilização de medicamentos. C: primeiro sugrupo da ATC, refere-se ao grupo terapêutico.

Não houve associação entre número de medicações prescritas, número de PPI e ocorrência de polifarmácia com idade ou gênero. Também não se correlacionaram PPI, número de medicamentos prescritos e ocorrência de polifarmacoterapia.

\section{DISCUSSÃO}

A frequência dos pacientes que receberam pelo menos uma prescrição inadequada foi significativamente superior às prevalências encontradas em outros estudos envolvendo pacientes idosos hospitalizados, entre 14\% e $21 \% .^{14-16}$

A maior taxa de prevalência de prescrições inapropriadas encontrada na literatura foi de $34 \%$, em um estudo com pacientes idosos atendidos em unidade geriátrica de urgência de hospital universitário na Irlanda, com idade média de 80,3 (+/- 6,1) anos, ou seja, de idade mais avançada que a dos pacientes do presente estudo $(70,4+/-7,3){ }^{1}$ Em outro estudo, encontrou-se prevalência de $32 \%$ em amostra constituída por idosos em estado grave, também na Irlanda, mas em enfermarias de medicina geral. ${ }^{17}$

Entre as categorias etárias de 70 a 79 anos e de 80 anos ou mais, o percentual de pacientes que recebeu prescrição inapropriada foi semelhante, embora os percentuais de 43 e 38\% sejam elevados, considerando que pacientes mais velhos têm maior tendência a apresentar os efeitos adversos dos medicamentos que não deveriam ser prescritos.

É preciso considerar, entretanto, que algumas das listas de PPI foram propostas por pesquisadores da França, Canadá e Estados Unidos. Estas não devem ser consideradas absolutas, sendo necessário estudo da 
farmacoepidemiologia de cada país para predizer quais os medicamentos considerados impróprios de acordo com o perfil de idosos e características de cada região. ${ }^{3}$ Contudo, o Critério de Beers é considerado o método mais utilizado para avaliar as características, com relação aos efeitos, dos medicamentos prescritos aos idosos.

Quanto à prevalência de polifarmacoterapia, a taxa encontrada foi um pouco inferior às prevalências relatadas em outros trabalhos envolvendo idosos hospitalizados: $62,3 \% \mathrm{em}$ um estudo feito em outro país, e de $85,7 \%$, em um estudo brasileiro, ${ }^{18}$ tendo sido estes estudos também realizados em serviços terciários, onde são internados pacientes portadores de múltiplas doenças crônicas, ou seja, a maioria destes pacientes realmente necessita usar múltiplas medicações. ${ }^{15}$

A chamada "polifarmácia", justificada em muitos casos no paciente idoso, é frequentemente desproporcional a seus problemas crônicos e pode ocasionar importante deterioração da sua saúde. Assim, em um serviço de atenção terciária, a averiguação clínica desta informação medicamentosa deveria ser feita sistematicamente. É importante destacar que, além da definição de polifarmácia como o uso de cinco ou mais medicamentos por dia, há outras definições que levam em conta o tempo de uso das medicações ou se o fármaco está sendo utilizado para controlar o efeito adverso de outro, não havendo, portanto, consenso universal sobre a definição desta condição. ${ }^{?}$

A distribuição de medicamentos por grupo terapêutico diferiu dos dados encontrados por outros autores, que verificaram maior consumo de agentes cardiovasculares e analgésicos/antiinflamatórios. ${ }^{19,20}$

A observação de maior frequência de uso de medicamentos do grupo do sistema digestório pode ter se associado ao fato de que a classe de doenças mais frequente foi a relacionada a este sistema na amostra deste estudo. No entanto, outro trabalho anterior realizado nas mesmas enfermarias de clínica médica do mesmo serviço (HULW) também demonstrou que esta classe de medicamentos é uma das mais frequentemente prescritas para os pacientes de todas as faixas etárias. ${ }^{21} \mathrm{~A}$ amostra do referido estudo foi constituída por 140 pacientes, com idade média inferior à da presente pesquisa $(47,3$ +/- 17,3 anos).

Pode-se afirmar que houve excesso de prescrições de antiulcerosos e antieméticos na amostra estudada, fugindo do padrão de prescrição medicamentosa do idoso, provavelmente relacionadas à prescrição irracional. Estes dados podem estar relacionados com a prescrição irracional de medicamentos nas enfermarias onde se realizaram estes estudos, com prescrição sistemática e excessiva de bloqueadores $\mathrm{H} 2$, provavelmente para prevenir úlceras de estresse, por exemplo. A porcentagem de prescrições com presença de antibióticos $(25,3 \%)$ está de acordo com o sugerido pela International Network for the Rational Use of Drug, em estudo realizado em 12 países indicando valores entre 20 a 30\% como aceitáveis. ${ }^{22}$

O achado do presente estudo, que indica não haver associação entre polifarmácia e PPI, diverge da hipótese de Johnell e Fastbom, que acreditam que os multiusuários de medicamentos tinham maior risco de usar PPI, embora tenham observado que os multiusuários parecem ter menor probabilidade de apresentar interações medicamentosas potencialmente sérias. ${ }^{23}$ Gallagher et al. também verificaram que quando pacientes idosos hospitalizados usam mais de cinco medicamentos simultaneamente, aumenta bastante a probabilidade de PPI. ${ }^{17}$

No Brasil, estudos e aplicações sobre esta temática são escassos. Informações sobre o padrão de uso de medicamentos entre idosos brasileiros são provenientes de estudos conduzidos nos municípios do Rio de Janeiro, Fortaleza e em Minas Gerais. ${ }^{6,20,24-26}$ Apenas dois destes estudos investigaram aspectos relacionados à qualidade da farmacoterapia.

No campo da investigação, é preciso conhecer o perfil dos usuários idosos, segundo as diferentes realidades sociais, geográficas e sanitárias; avaliar a qualidade do conjunto dos produtos 
consumidos e, ao mesmo tempo, identificar os principais preditores do uso irracional.

\section{CONCLUSÕES}

Conclui-se que houve alta prevalência de PPI e excesso de prescrições de antiulcerosos e antieméticos na amostra estudada, fugindo do padrão de prescrição medicamentosa do idoso, provavelmente relacionadas à prescrição irracional. Tais resultados indicam que maior atenção deve ser dada à prescrição para pacientes idosos por médicos residentes em enfermarias de clínica geral e que conteúdos de saúde do idoso devem ser enfocados na programação de educação continuada da residência em clínica médica.

\section{REFERÊNCIAS}

1. Barry PJ, O'Keefe N, O'Connor KA, O'Mahony D. Inappropriate prescribing in the elderly: a comparison of the Beers criteria and the improved prescribing in the elderly tool (IPET) in acutely ill elderly hospitalized patients. J Clin Pharm Ther 2006; 31(6):617-26.

2. Ribeiro AQ, Araújo CMC, Acurcio FA, Magalhães SMS, Chaimowicz F. Qualidade do uso de medicamentos por idosos: uma revisão dos métodos de avaliação disponíveis. Ciência \& Saúde Coletiva 2005; 10 (4): 1037-45.

3. Quinalha JV, Correr CJ. Instrumentos para avaliação da farmacoterapia do idoso: Uma revisão. Revista brasileira de geriatria e gerontologia.2010; 13(3): 487-500.

4. Passarelli MCG. Reações adversas a medicamentos em uma população idosa [tese]. São Paulo: Faculdade de Medicina, Universidade de São Paulo; 2005.

5. Bernstein LR, Folkman S, Lazarus RS.

Characterization of the use and misuse of medications by an elderly, ambulatory population. Med Care 1989; 27(6):654-63.

6. Rozenfeld S. Reações adversas aos medicamentos na terceira idade: as quedas em mulheres como iatrogenia farmacoterapêutica [tese]. Rio de Janeiro: Instituto de Medicina Social, Universidade Estadual do Rio de Janeiro; 1997.
Posteriormente, deveriam ser realizados novos estudos no nosso serviço, para avaliar as causas do padrão prescritivo dos pacientes idosos, no sentido de avaliar a racionalidade dessa prática. A difusão do conceito de uso inapropriado mediante educação continuada para médicos generalistas poderia facilitar a adoção dos critérios de Beers, ainda não conhecidos por toda a comunidade médica.

\section{AGRADECIMENTO}

Agradecemos aos médicos ex-residentes do HULW, Roberto Luiz Pereira Matias e Renato Ramos, pela colaboração na coleta dos dados desta pesquisa.
7. Girotto E, Da Silva PV. A prescrição de medicamentos em um município do Norte do Paraná. Rev Bras Epidemiol. 2006; 9(2): 226-34.

8. Sousa-Muñoz RL, Oliveira SDG, Goncalves CEF. Avaliação do uso de medicamentos pela clientela idosa à admissão nas enfermarias de clínica médica do HULW/UFPB. In: Simpósio Internacional de Medicamentos; 2002; João Pessoa.

9. Corsonello A, Pedone C, Corica F, Incalzi RA. Polypharmacy in elderly patients at discharge from the acute care hospital. Ther Clin Risk Manag 2007; 3 (1): 197-203.

10. Fick DM, Cooper JW, Wade WE, Waller JL, Maclean JR, Beers MH. Updating the Beers criteria for potentially inappropriate medication use in older adults: results of a US consensus panel of experts. Arch Intern Med 2003. 163(22): 2716-24.

11. Brasil. Ministério da Saúde Secretaria de Políticas de Saúde. Política Nacional de saúde do idoso (PNSI). Rev Saúde Pública. 1999; 33 (6):67-70.

12. Paes P. Projeto de Lei do Senado PLS n0 95/2005. Subcomissão Permanente de Promoção, Acompanhamento e Defesa da Saúde Disponível em: URL: http://www.senado.gov.br/web/senador/ papaleopaes/impressao/projetos/pls95_2005.htm.

13. Centro Brasileiro de Classificação de Doenças. Classificação de Doenças em Português. 
São Paulo: Faculdade de Saúde Pública da Universidade de São Paulo; 1993.

14. Fialová D, Topinková E, Gambassi G, FinneSoveri H, Jónsson PV, Carpenter I, et al. Potentially inappropriate medication use among elderly home care patients in Europe. JAMA 2005; 293 (11):1348-58.

15. Wawruch M, Zikavska M, Wsolova L, Kuzelova M, Tisonova J, Gajdosik J, et al. Polypharmacy in elderly hospitalised patients in Slovakia. Pharm World Sci. 2008; 30 (3): 235-42.

16. Onder G, Landi F, Cesari M, Gambassi G, Carbonin $\mathrm{P}$, Bernabei R, et al. Inappropriate medication use among hospitalized older adults in Italy: results from the Italian Group of Pharmacoepidemiology in the elderly. Eur J Clin Pharmacol. 2003; 59 (2): 157-62.

17. Gallagher PF, Barry PJ, Ryan C, Hartigan I, O'Mahony D. Inappropriate prescribing in an acutely ill population of elderly patients as determined by Beers' Criteria. Age Ageing. 2008; 37 (1): 96-101.

18. Loyola Filho AI, Uchoa E, Firmo JOA, LimaCosta MF. Influência da renda na associação entre disfunção cognitiva e polifarmácia: Projeto Bambuí. Rev Saúde Pública 2008; 42 (1): 89-99.

19. Marin MJ, Cecílio LC, Perez AE, Santella F, Silva CB, Gonçalves Filho JR, et al. Use of medicines by the elderly in a Family Health Program unit in Brazil. Cad Saúde Pública. 2008; 24(7): 1545-55.
20. Mosegui GBG. Avaliação da qualidade do uso de medicamentos em idosos [tese]. Rio de Janeiro; 1997.

21. Taguchi LC. Avaliação cognitiva breve no âmbito da medicina geral: Contribuição à semiologia do paciente internado em enfermarias de clínica médica. [monografia]. João Pessoa: Hospital Universitário Lauro Wanderley, Programa de Residência Médica, Universidade Federal da Paraíba; 2008.

22. Hogerzeil HV, Bimo, Ross-Degnan D, Laing RO, Ofori-Adjei D, Santoso B, et al. Field tests for rational drug use in twelve developing countries. Lancet. 1993; 342 (8884): 1408-10.

23. Johnell K, Fastbom J. Multi-dose drug dispensing and inappropriate drug use: A nationwide registerbased study of over 700,000 elderly. Scand J Prim Health Care. 2008; 26(2): 86-91.

24. Miralles MA, Kimberlin CL. Perceived access to care and medication use among ambulatory elderly in Rio de Janeiro, Brazil. Soc Sci Med. 2005; 46 (3): 345-55.

25. Chaimowicz F. A saúde dos idosos brasileiros às vésperas do século XXI: problemas, projeções e alternativas. Rev Saúde Pública. 1997; 31(2):184-200.

26. Coelho Filho JM, Marcopito LF, Castelo A. Perfil de utilização de medicamentos por idosos em área urbana do Nordeste do Brasil. Rev Saúde Pública. 2004; 38 (4): 557-64. 